effects are observed in the proximal portion of the contralateral coronary artery and thus are not selective when administered as in our protocol.

Hackett et al $^{1}$ administered intracoronary ergonovine to a maximum cumulative dose of $50 \mu \mathrm{g}$ in 6 patients with variant angina and in 9 patients similar to the group included in the current study. In the latter group, intracoronary ergonovine produced vasoconstriction of a similar magnitude as seen in our study; however, proximal, mid- and distal segment responses were not compared and the contralateral coronary artery was not measured. Fournier et $\mathrm{al}^{2}$ administered single or repeated intracoronary bolus doses $(25 \mu \mathrm{g})$ of ergonovine to 108 patients with normal coronary angiography. Again, ergonovine caused vasoconstriction but no contralateral coronary artery effects were measured.

In general, our data show a dose-dependent ergonovine-mediated vasoconstriction, although in 1 patient all right coronary artery segments revealed vasodilation after $10 \mu \mathrm{g}$ (Figure 2), which was overcome with higher doses of ergonovine. Mohri et $\mathrm{al}^{4}$ described a similar case, in which a dilator response of both the left and right coronary arteries was observed with $20 \mu \mathrm{g}$ intracoronary ergonovine followed by a constrictor response when an additional $35 \mu \mathrm{g}$ intracoronary ergonovine was administered. These results are similar to observations of the effect of intravenously administered ergonovine on coronary arterial diameter in dogs. This response has been shown to be biphasic, in the form of an initial dilating action followed by a constricting action. ${ }^{5}$

The magnitude of mean proximal right coronary artery constriction (69\%) after administration of $60 \mu \mathrm{g}$ ergonovine into the left main coronary artery was greater than the effect seen when ergonovine was administered directly into the right coronary artery (44\%). When ergonovine was administered into the right coronary artery, a $19 \%$ mean decrease in left main coronary artery area was observed, whereas a $33 \%$ decrease was seen when ergonovine was given directly into the left main. Although these measurements cannot be directly compared because they were obtained from different patients, the magnitude of the responses observed in the proximal contralateral artery is impressive. The mechanism by which intracoronary ergonovine affects the contralateral coronary artery in its most proximal segment cannot be deduced from this observational study. One obvious possibility includes spilling of ergonovine during systole into the contralateral sinus despite precautions taken to prevent this by careful catheter placement and slow injection. Other potential mechanisms include recirculation of ergonovine, preresistance vessel or collateral shunting of blood or local reflexes. In any event, the assumption that one is safely getting a selective unilateral coronary vasoconstrictor effect during intracoronary ergonovine administration is not supported by the data presented herein. Whether this represents a cause for concern clinically must await further studies. Contralateral coronary angiograms should probably always be recorded after intracoronary administration of ergonovine so as not to miss important coronary vasoconstriction that may occur.

1. Hackett D, Larkin S, Chierchia S, Davies G, Kaski JC, Maseri A. Induction of coronary artery spasm by a direct local action of crgonovinc. Circulation 1987;75:577-582.

2. Fournier JA, Cortacero JAP, Tura A, Hernandez-Aparicio C, Granado C, Vallejo J. Effects of intracoronary injection of ergonovine on angiographic normal coronary arteries: study of 108 consecutive patients. Clin Cardiol 1989;12:561 568

3. Silver KH, Buczec JA, Esser PD, Nichols AB. Quantitative analysis of coronary angiograms by microprocessor cine-videodensitometry. Cathet Cardiovasc Diagn 1987;13:291-300

4. Mohri M, Okamatsu S, Nakamura M. Case report: biphasic response of coronary arterial diameter to intracoronary ergonovine. Clin Cardiol 1988; 11:710-714.

5. Holtz J, Held W, Sommer O, Kuchne G, Bassenge E. Ergonovine-induced constrictions of epicardial coronary arteries in conscious dogs: alpha-adrenocep tors are not involved. Basic Res Cardiol 1982;77:278-283.

\title{
Hypercholesterolemia After Cardiac Transplantation in Children
}

\author{
Karen Uzark, RN, PhD, Dennis Crowley, MD, Louise Callow, MSN, and Edward Bove, MD
}

S ince the 1980 s a steadily increasing number of children have undergone cardiac transplantation. Although graft rejection and complications of immunosuppressive therapy are the leading causes of death in children and adults after cardiac transplantation, coronary atherosclerosis is a significant cause of late mortality. Our first pediatric heart transplant patient died suddenly 17 months after transplantation at 3 years of age with severe coronary atherosclerosis. Transplant coronary atherosclerosis may be the result of immune endothelial injury, with a response characterized by proliferation of

From the University of Michigan Medical Center, Division of Pediatric Cardiology and Division of Thoracic Surgery, Room F1 126, Box 0204 , 1500 East Medical Center Drive, Ann Arbur, Michigan 48109-0204 Manuscript received April 23, 1990; revised manuscript received and accepted July 18, 1990. myointimal cells, thrombus formation, and ultimate intraarterial deposition of lipid material. ${ }^{3-5}$ The development of atherosclerosis in transplanted hearts may be enhanced by the presence of hyperlipidemia, both hypertriglyceridemia and hypercholesterolemia. We measured serial lipid levels in 14 children after heart transplantation to assess the prevalence of lipid abnormalities and the usefulness of niacin therapy in children with significant hypercholesterolemia after transplantation.

The study sample comprised 14 children (aged 6 months to 16 years [mean 8]) who underwent cardiac transplantation. There were 5 girls and 9 boys. Eight of 14 children $(57 \%$ ) received transplants for cardiomyopathy, 1 restrictive, 7 of the dilated type, and 1 caused by anthracyclines. The remaining 6 children had severe ventricular dysfunction with congenital heart disease-4 with complex cyanotic heart disease, 1 with severe aortic 


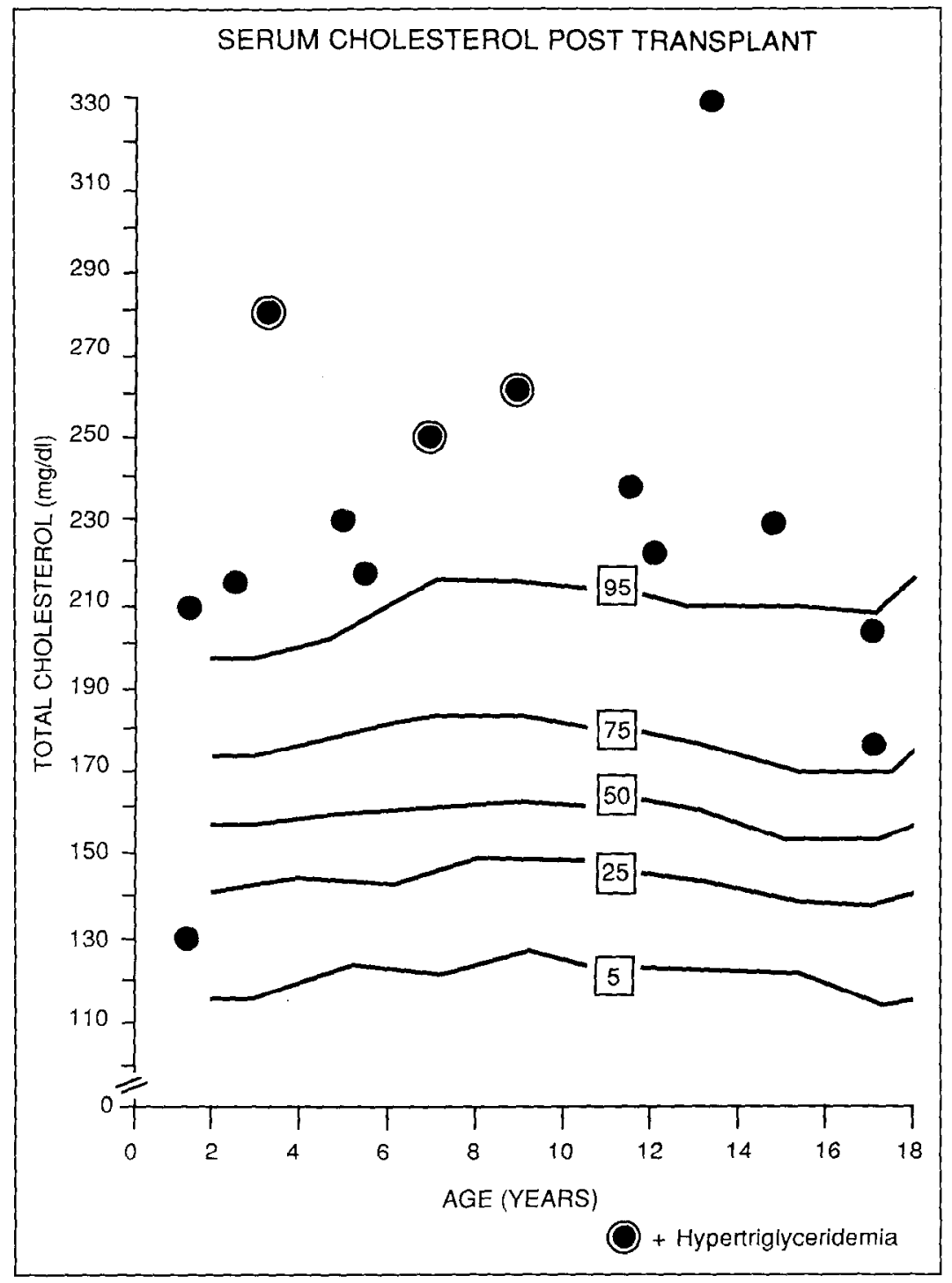

FIGURE 1. Serum total cholesterol in patients (closed bullets) after cardiac trans. plantation by age as compared to Bogalusa Heart Study ${ }^{8}$ data. Note 3 patients who also have hypertriglyceridemia.

stenosis, and 1 with hypoplastic left heart syndrome. Five patients had undergone previous palliative surgical procedures. The weight for height at the time of assessment after transplantation was greater than the 75th percentile in 6 of 14 patients. All children were maintained on daily prednisone, 0.2 to 0.7 (mean 0.4) $\mathrm{mg} / \mathrm{kg}$ and on cyclosporine, 5 to 163 (mean 39) $\mathrm{mg} / \mathrm{kg} /$ day. In addition, azathioprine, 0.5 to $2.4 \mathrm{mg} / \mathrm{kg} /$ day (mean 1.4), was used in 10 of 14 patients. All patients had been discharged after transplantation on a low cholesterol diet. Lipid profiles were obtained at 2- to 6-month intervals in association with endomyocardial biopsies. Serum cholesterol and triglyceride concentrations were measured by enzymatic oxidase methods.

Within 2 to 12 months after cardiac transplantation (mean 8.5), 12 of 14 children (86\%) had a total serum cholesterol $>90$ th percentile for age by Bogalusa criteria (Figure 1) and $>190 \mathrm{mg} / \mathrm{dl}$. Only one child, our youngest at transplantation, had a total cholesterol below the 75 th percentile for age. Three of 10 patients also had hypertriglyceridemia. High-density lipoprotein cholesterol ranged from 23 to $65 \mathrm{mg} / \mathrm{dl}$ (mean 48 ).
Whereas previous studies by others have suggested that hyperlipidemia in patients after heart and kidney transplantation is related in some way to the effects of therapy with corticosteroids ${ }^{6}$ and cyclosporine, ${ }^{7}$ no sig. nificant correlations were found between serum cholesterol and the daily prednisone dose $(r=-0.16)$, daily cyclosporine dose $(r=-0.04)$, or the relative body weights of our pediatric patients. The lack of correlation between prednisone dose or cyclosporine dose and serum cholesterol levels in the present study may be due to the small sample size or to insufficient sample variation. While 6 of 14 children in our study had excess weight gain after transplantation, significant hypercholesterolemia was also noted in children who did not gain weight.

Niacin therapy was instituted in 5 patients with cholesterol levels persistently $>210 \mathrm{mg} / \mathrm{dl}$, with a mean cholesterol level in these children of $265 \mathrm{mg} / \mathrm{dl}$. Niacin dosage ranged from $50 \mathrm{mg}$ twice a day in the youngest patient ( 3 years old) to $500 \mathrm{mg}$ twice a day in 2 patients $(>9$ years). In all 5 children receiving niacin, there was $a$ significant decrease in serum cholesterol $(t=6.55 ; p$ $<0.01$ ), with a mean reduction in serum cholesterol of 73 
FIGURE 2. Serum cholesterol change with time after initiation of niacin therapy (RX).

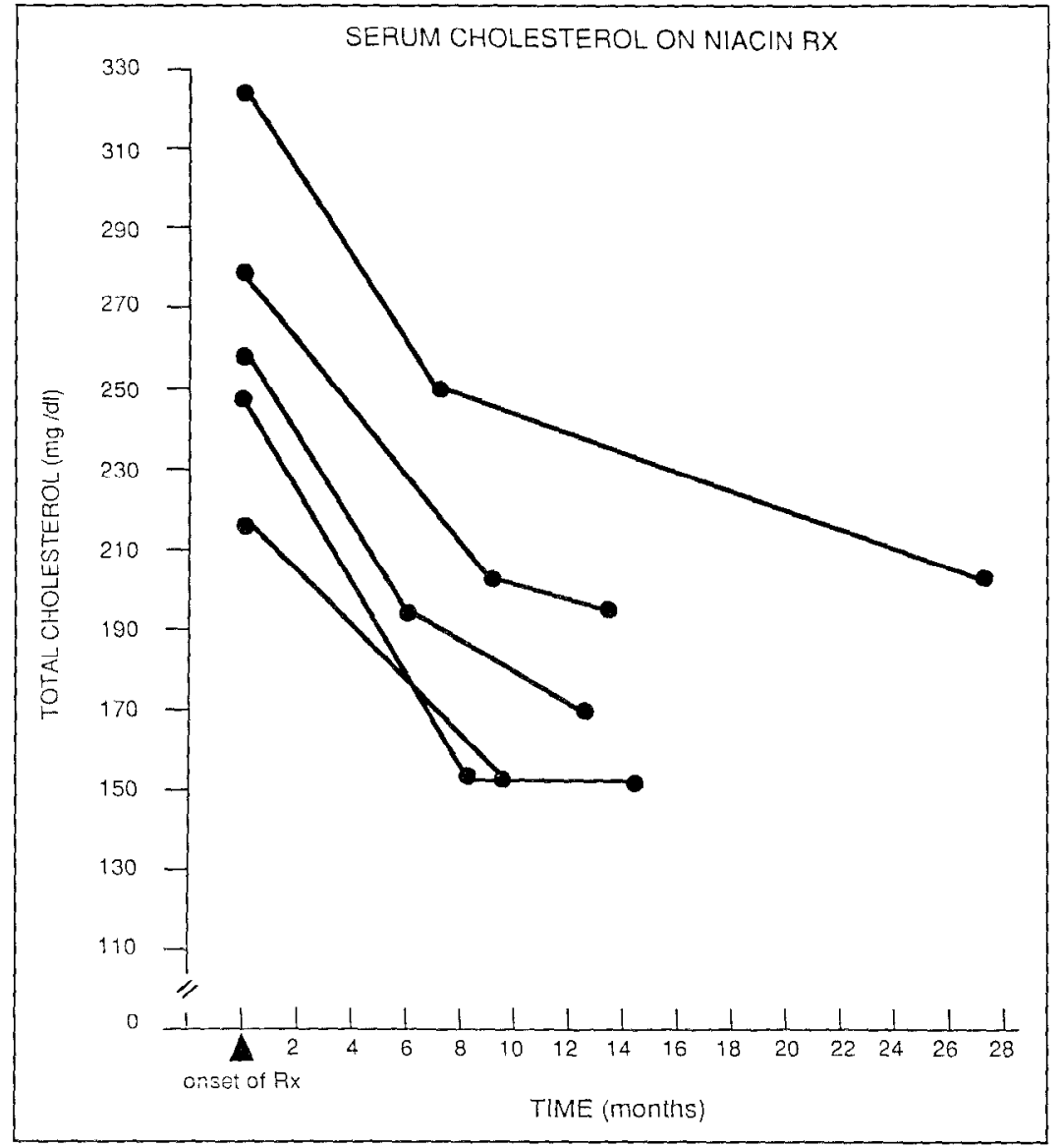

$\mathrm{mg} / \mathrm{dl}$ (Figure 2). Only 1 child had side effects, mainly flushing, which resolved with the addition of an aspirin with his evening niacin dose. There were no significant changes in cyclosporine levels with niacin therapy.

We conclude that hyperlipidemia, specifically hypercholesterolemia, is a common problem in children $>2$ years of age after cardiac transplantation. Our results are consistent with previous studies that have shown an increased prevalence of hyperlipidemia in adult transplant recipients. ${ }^{3}$ Atherosclerosis begins early in life, and the results of the Bogalusa Heart Study demonstrate that the extent of aortic fatty streaks is very strongly related to serum cholesterol levels. ${ }^{8}$ In transplanted hearts, accelerated coronary atherosclerosis has been a documented problem. ${ }^{1-5}$ Most pediatric heart transplant recipients may be at increased risk for development of coronary artery disease, not only because of elevated serum cholesterol levels, but also because of hypertension associated with cyclosporine therapy and with weight gain or obesity after transplantation, which may further increase their cardiovascular risk. Unfortunately, treatment of hyperlipidemia is limited by the availability of effective, welltolerated drugs that do not adversely affect immunosuppressive therapy, as well as by the limited efficacy of dietary therapy. Niacin therapy seems to be a safe and effective way of treating posttransplant hyperlipidemia in these children.

We recommend periodic monitoring of lipids in children within 2 months of transplantation. Furthermore, in children over 2 years of age with significant hyperlipidemia (elevated cholesterol, triglycerides, or both), we recommend niacin therapy, beginning with small doses (50 $\mathrm{mg}$ ) and slowly increasing the dose until the desired effect is achieved, while monitoring for potential side effects. Further research is needed to establish the association of hyperlipidemia after heart transplantation with graft atherosclerosis, and to assess the relation between lipid-lowering therapy and the development of coronary atherosclerosis in patients of all ages after heart transplantation.

1. Pennock $J L$, Oyer PE, Reitz BA. Cardiac transplantation in perspective for the future. J Thorac Cardiovasc Surg 1982;83:168-177

2. Addonizio LJ, Hsu DT, Smith CR, Rose EA. Late complications in pediatric cardiac transplant recipients (abstr). Circulation 1989;80(suppl):4.

3. Becker DM, Chamberlain B, Swank R, Hegewald MG, Girardet R, Baughman KL, Kwiterovich PO, Pearson TA, Ettinger WH, Renlund D. Relationship between corticosteroid exposure and plasma lipid levcls in heart transplant recipients. Am J Med 1988;85:632-638.

4. Hess MJ, Hastillo A, Mohanakuman T, Cowley M, Vetrovac G, Szentpetery $\mathrm{S}$, Wolfgang TC, Lower RR. Accelerated athcrosclerosis in cardiac transplantation: role of cytotoxic B-cell antibodies and hyperlipidemia. Circulation 1983;68 (suppl):94-101

5. Lurie KG, Billingham ME, Jamieson SW, Harrison DC, Reitz BA. Pathogenesis and prevention of graft atherosclerosis in an experimental heart transplant model. Transplantation 1981;31:41-47.

6. Ibels LS, Alfrey AC, Weil R. Hyperlipidemia in adult, pediatric and diabetic renal transplant recipients. Am J Med 1978;64;634-642.

7. Harris KP, Russel GL, Parvin SD, Vertch PS, Walis J. Alterations in lipid and carbohydrate metabolism attributable to cyclosporine $A$ in renal transplant recipients. Br Med J 1986;292:16.

8. Berenson GS, ed. Causation of Cardiovascular Risk Factors in Children-Perspectives on Cardiovascular Risk in Early Life. New York: Raven Press, $1986: 40,346$. 